\title{
Introduction to the TPLP Special Issue from the 16th European Conference on Logics in Artificial Intelligence (JELIA 2019)
}

\author{
FRANCESCO CALIMERI, MARCO MANNA and SIMONA PERRI \\ Department of Mathematics and Computer Science, University of Calabria, Italy \\ (e-mails: francesco.calimeri@unical.it, marco.manna@unical.it, simona.perri@unical.it)
}

submitted 18 May 2021; revised 12 July 2021; accepted 13 July 2021

This volume contains a selection of the best papers presented at the 16th European Conference on Logics in Artificial Intelligence (JELIA 2019) held on May 7-11, 2019 in Rende (Cosenza), Italy; this edition was organized by the Artificial Intelligence Group of the Department of Mathematics and Computer Science at the University of Calabria.

The European Conference on Logics in Artificial Intelligence (or Journes Europennes sur la Logique en Intelligence Artificielle - JELIA) began back in 1988, as a workshop, in response to the need for a European forum for the discussion of emerging work in this field. Since then, JELIA has been organized biennially, with proceedings published in the Springer-Verlag series Lecture Notes in Artificial Intelligence. Previous meetings took place in Roscoff, France (1988), Amsterdam, the Netherlands (1990), Berlin, Germany (1992), York, UK (1994), vora, Portugal (1996), Dagstuhl, Germany (1998), Mlaga, Spain (2000), Cosenza, Italy (2002), Lisbon, Portugal (2004), Liverpool, UK (2006), Dresden, Germany (2008), Helsinki, Finland (2010), Toulouse, France (2012), and Larnaca, Cyprus (2016).

JELIA always aimed at bringing together active researchers interested in all aspects concerning the use of logics in artificial intelligence, in order to foster discussions about current research, results, problems, and applications of both theoretical and practical nature; furthermore, it helps at fostering links and facilitating cross-fertilization of ideas among researchers from various disciplines, from academia and industry, and between theoreticians and practitioners. The scientific community has been increasingly showing interest in JELIA, which during the years featured a growing participation of researchers from outside Europe and a very high overall technical quality of contributions. This has allowed JELIA to become a major biennial forum and a reference for the discussion of approaches, especially logic-based, to artificial intelligence.

JELIA 2019 received 126 submissions in 2 different formats (long and short papers); eventually, 50 papers, 40 long and 10 short ones, were accepted for presentation. The present special issue of the Journal of Theory and Practice of Logic Programming consists of a small set of works carefully selected from the 40 long papers. After the first shortlisting of the submissions featuring the highest scores as assigned by the original reviewers from the Program Committee, and a thorough examination of all reviews and the paper contents, a restricted selection was identified for the actual invitation. As a 
result of a two-round review process, during which each submission was reviewed by three referees, four papers have been accepted to be included in the issue.

The first work, "The Probabilistic Description Logic BALC" by Leonard Botha, Thomas Meyer and Rafael Pealoza, introduces and discusses BALC, a Bayesian extension of the Description Logic ALC. The second paper, "A Qualitative Theory of Cognitive Attitudes and their Change" by Emiliano Lorini, presents and characterizes a general logical framework for reasoning about agents cognitive attitudes of both epistemic type and motivational type. The third work, entitled "Exploring the Jungle of Intuitionistic Temporal Logics", by Joseph Boudou, Martín Diéguez, David Fernández-Duque and Philip Kremer, proposes a set of axiomatic systems for intuitionistic linear temporal logic. Finally, the fourth paper, "Completing the Picture: Complexity of Graded Modal Logics with Converse" by Bartosz Bednarczyk, Emanuel Kieroski and Piotr Witkowski, presents and discusses a complete classification of the graded modal language with graded converse modalities.

We would like to thank all the expert reviewers: their valuable work throughout the complex multistage reviewing process has been crucial for the production of the present issue; moreover, we want to express our gratitude and appreciation to the staff of Theory and Practice of Logic Programming, and in particular to the editor-in-chief Mirek Truszczynski, for his continued help and kind support. 